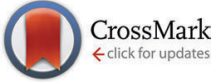

Cite this: J. Mater. Chem. B, 2015, 3, 5010

Received 9th January 2015, Accepted 9th February 2015

DOI: $10.1039 / c 5 t b 00053 j$

www.rsc.org/MaterialsB

\title{
Poly[3,4-ethylene dioxythiophene (EDOT)-co- 1,3,5-tri[2-(3,4-ethylene dioxythienyl)]-benzene (EPh)] copolymers (PEDOT-co-EPh): optical, electrochemical and mechanical properties
}

\author{
Liangqi Ouyang, ${ }^{a}$ Chin-chen Kuo, ${ }^{a}$ Brendan Farrell, ${ }^{b}$ Sheevangi Pathak, ${ }^{c}$ Bin Wei, ${ }^{a}$ \\ Jing $\mathrm{Qu}^{\mathrm{a}}$ and David C. Martin*ab
}

\begin{abstract}
PEDOT-CO-EPh copolymers with systematic variations in composition were prepared by electrochemical polymerization from mixed monomer solutions in acetonitrile. The EPh monomer is a trifunctional crosslinking agent with three EDOTs around a central benzene ring. With increasing EPh content, the color of the copolymers changed from blue to yellow to red due to decreased absorption in the near infrared (IR) spectrum and increased absorption in the visible spectrum. The surface morphology changed from rough and nanofibrillar to more smooth with rounded bumps. The electrical transport properties dramatically decreased with increasing EPh content, resulting in coatings that either substantially lowered the impedance of the electrode (at the lowest EPh content), leave the impedance nearly unchanged (near $1 \% \mathrm{EPh}$ ), or significantly increase the impedance (at $1 \%$ and above). The mechanical properties of the films were substantially improved with EPh content, with the $0.5 \%$ EPh films showing an estimated $5 \times$ improvement in modulus measured by AFM nanoindentation. The PEDOT-CO-EPh copolymer films were all shown to be non-cytotoxic toward and promote the neurite outgrowth of PC12 cells. Given these results, we expect that the films of most interest for neural interface applications will be those with improved mechanical properties that maintain the improved charge transport performance (with 1\% EPh and below).
\end{abstract}

\section{Introduction}

Conducting polymers such as the functionalized polythiophene poly(3,4-ethylene dioxythiophene) (PEDOT) have many useful qualities including versatile organic chemistry, simple processing methods, excellent biocompatibility and the ability to switch between different oxidation states. They are also known to facilitate both ion and electron transport. All of these characteristics make them attractive materials for tissue engineering, ${ }^{1,2}$ biosensing $^{3-5}$ and neural interfaces. ${ }^{6,7}$ In these applications, conducting polymers are intended to directly interface with electroactive cells such as neural tissue. Electric charge can be delivered to stimulate tissue $^{8}$ or to modulate cell growth. ${ }^{1,9}$ Cellular activities can also be recorded with high levels of sensitivity. ${ }^{10}$

Conducting polymers can be readily synthesized through electrochemical polymerization. Typically, current is delivered into a monomer solution, and the monomer is electrochemically oxidized onto the anode. This method has proven to be particularly

\footnotetext{
${ }^{a}$ The University of Delaware, Materials Science and Engineering, USA. E-mail:milty@udel.edu

${ }^{b}$ The University of Delaware, Biomedical Engineering, USA

${ }^{c}$ Pennsylvania State University, Materials Science and Engineering, USA
}

useful for the coating of neural microelectrodes. When one of the most stable conducting polymers, poly(3,4-ethylenedioxythiophene) (PEDOT), is deposited, it forms bumpy and nano-fibrous morphologies that dramatically increase the effective surface area of the electrodes. ${ }^{11}$ After coating with PEDOT, the impedance of the electrode in the biologically important frequency range of $1 \mathrm{kHz}$ and lower is significantly decreased, typically by two to three orders of magnitude. ${ }^{12}$ The charge storage capacity (CSC) of PEDOT-coated electrodes is also greatly enhanced. Much faster and lower voltage responses are observed when delivering stimulation charge through PEDOT. ${ }^{13}$

Another advantage of employing conducting polymers as direct interfacing materials with tissues is that these are relatively soft materials. The typical young's modulus of PEDOT is around $2 \mathrm{GPa}$, which is significantly lower than that of inorganic metals or semiconductors $(\sim 100 \mathrm{GPa}){ }^{14}$ By using various polyanions as dopants, the stiffness of the polymer coating can be further decreased. ${ }^{15,16}$ The soft and flexible polymer surface allows for better contact with the cells. It has been demonstrated that highly conformal neural electrodes can be made with PEDOT as the conducting material. ${ }^{17}$ Using a softer electrode material decreases the mechanical properties mismatch between the hard electrode and the soft tissue, ${ }^{18}$ 
which is expected to be beneficial for mitigating the chronic foreign body response. ${ }^{19,20}$ Recently, we showed that PEDOT could even be directly deposited into living rat hippocampus as a potential strategy for creating locally conductive pathways between implanted electrodes and the surrounding neural tissue. ${ }^{21}$

However, depending on the chemistry and method of preparation, conducting polymer films can be relatively fragile. PEDOT films have been found to crack or delaminate from solid substrates under mechanical deformation. Sterilization and chronic stimulation can comprise the film integrity. ${ }^{22,23}$ When interfacing with peripheral nerves, electrodes are likely to be subjected to particularly severe strains due to chronic muscle movements. ${ }^{24}$ Cracking and delamination of the conducting polymer coating may not only decrease the performance of the material itself, but could also leave behind fractions of the film in the tissue. Although a number of studies have demonstrated that PEDOT itself is not cytotoxic, ${ }^{25}$ the impact of potential fragments of PEDOT remains to be studied in detail. It is therefore of interest to more carefully examine the mechanical performance of PEDOT, and to develop potential methods for improving its stiffness, strength, and durability.

Several strategies have been reported for improving the mechanical durability of PEDOT films. For example, composite materials were made by blending or dispersing PEDOT into $\mathrm{PDMS}^{26}$ or $\mathrm{PVA}^{27}$ matrices. These soft polymer matrices provided mechanical support to PEDOT. However, as the content of these non-conducting matrix materials increased, the conductivity of the composites decreased dramatically. PEDOT was also deposited into a hydrogel network, where the soft and durable hydrogel served as a mechanical buffer. Inganas and coworkers chemically crosslinked the polyelectrolyte dopant PSS and found that the stability of PEDOT:PSS was substantially improved. ${ }^{28,29}$ Another method was to introduce an EDOT derivative consisting of two EDOT units linked through ethylene oxide units. ${ }^{30} \mathrm{CV}$ sweeps confirmed that this material had improved electrochemical stability. Despite these developments, the search for conducting polymer materials that are more mechanically robust remains an ongoing topic of interest.

In order to create more mechanically robust PEDOT coatings for the neural interfacing applications, we examined a strategy for chemically crosslinking the PEDOT into a network structure by introducing a branched comonomer during electrochemical synthesis. A conjugated 3-armed EDOT derivative, 1,3,5-tri[2(3,4-ethylenedioxythienyl)]-benzene (EPh), ${ }^{31,32}$ which consists of a benzene core surrounded by three EDOT moieties (Scheme 1), was used as the crosslinking agent. We felt that this crosslinker would improve mechanical properties of the PEDOT, and might be also able to preserve charge transport properties because of the potential for extended electron conjugation through the benzene ring in the core of the molecule (Fig. 1).

Crosslinked conjugated polymer networks have been investigated for engineering organic semiconductors with isotropic charge transport properties ${ }^{33}$ or for creating stable microporous structures. ${ }^{34}$ However, the potential application of crosslinked conjugated polymer networks as neural interfacing materials had yet to be studied. Here, we report studies of the optical, electrochemical,
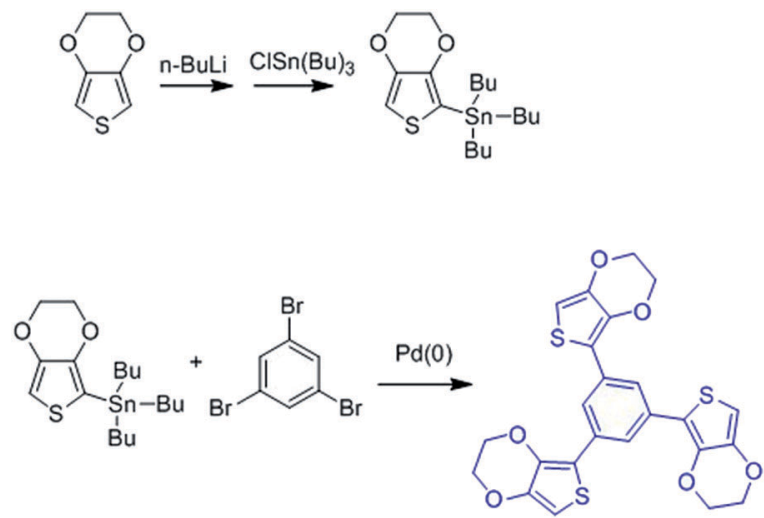

Scheme 1 Synthesis route of 1,3,5-tri[2-(3,4-ethylenedioxythienyl)]-benzene (EPh).

morphological, and mechanical properties of PEDOT-co-EPh copolymers with systematic variations in EPh content. We found that as the EPh content increased, the mechanical stiffness and strength of the copolymer films increased, but the electrochemical transport properties decreased. Adding EPh shifted the UV-vis absorption properties of the PEDOTco-EPh copolymers from the near infrared into the UV, making it possible to tune their color across the visible spectrum. The wetting angles of the PEDOT-co-EPh films did not change significantly with EPh content, and all of the copolymer films remained non-cytotoxic for PC-12 cells in vitro. The optimal films for neural interface applications are expected to be those that show significantly improved mechanical performance but still maintain high electrical transport properties, corresponding to $\mathrm{EPh}$ additions of around $1 \%$ or less.

\section{Experimental}

\section{Chemical synthesis}

The synthesis of 1,3,5-tri[2-(3,4-ethylenedioxythienyl)]-benzene (EPh) was adopted from Idzik et al. ${ }^{32,35}$ As shown in Scheme 1, first, $2 \mathrm{~g}$ EDOT (Sigma-Aldrich) was dissolved in $160 \mathrm{~mL}$ dry tetrahydrofuran (THF, Fisher Scientific) and then cooled down to $-78{ }^{\circ} \mathrm{C}$. After equilibrium, $10 \mathrm{~mL} 1.6 \mathrm{M} \mathrm{n}$-butyl lithium ( $n \mathrm{BuLi}$, Fisher Scientific) was added into the system dropwise. After reacting for 30 minutes, the temperature was increased to $47^{\circ} \mathrm{C}$ and $5.95 \mathrm{~g}$ tribultyl tin chloride (ClSn(Bu$)_{3}$, Sigma-Aldrich) was added into the solution dropwise and then the mixture was allowed to react for 24 hours. THF was removed by rotary evaporation, and the crude product was then dissolved in hexanes. The resultant mixture was then filtered and the filtrate was collected and the solvent was removed to yield slightly yellowish oil. EDOT-SnBu 3 was used without further purification.

For the synthesis of EPh, $0.63 \mathrm{~g}$ EDOT-SnBu, $0.460 \mathrm{~g}$ tetrakis(triphenylphosphine) palladium(0) $\left(\mathrm{PdPPh}_{3}\right.$, Sigma-Aldrich) and $2.846 \mathrm{~g}$ 1,3,5-tribromobenzene tetrakis(triphenylphosphine) palladium $(0)\left(\mathrm{PdP}\left(\mathrm{Ph}_{3}\right)\right)$ was dissolved in $150 \mathrm{~mL}$ anhydrous toluene under argon protection. The solution was stirred and refluxed under $110{ }^{\circ} \mathrm{C}$ for 48 hours. After being cooled to room temperature, water 


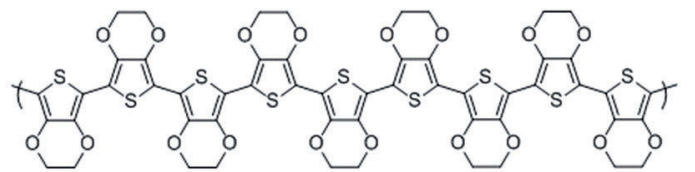

(a)

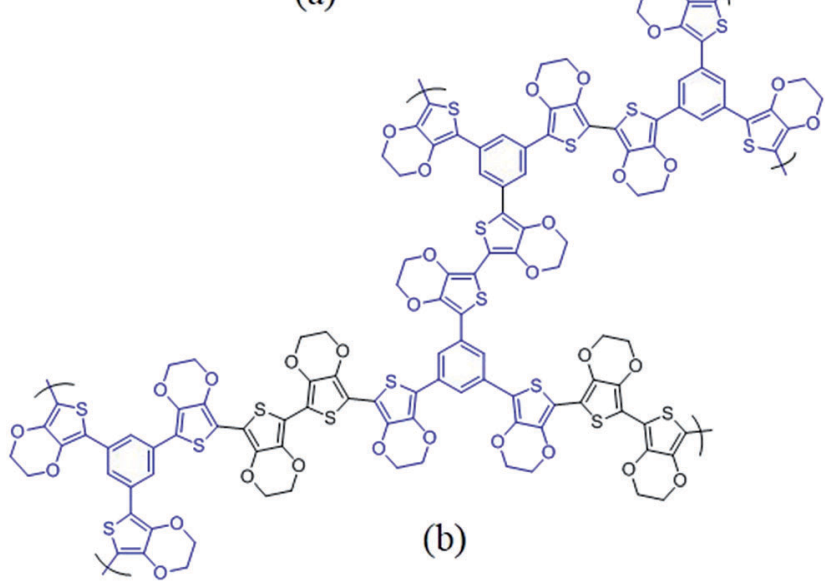

Fig. 1 (a) PEDOT and (b) EPh crosslinked PEDOT.

was added into the reaction mixture. The mixture was extracted with $\mathrm{CHCl}_{3}$ for 3 times. The organic layer was collected and washed 3 times with saturated $\mathrm{NaCl}$ solution and then DI water. It was then dried against anhydrous magnesium sulfate. The solvent was removed by rotary evaporation. The product was purified through a chromatographic column with hexane-ethyl acetate as eluent. The final product was a yellow solid $(400 \mathrm{MHz}$, $\left.\mathrm{CDC}_{13}\right):{ }^{1} \mathrm{H}$ NMR $\delta=7.92(\mathrm{~s}, 3 \mathrm{H}) ; 6.34(\mathrm{~s}, 3 \mathrm{H}) ; 4.36-4.34(\mathrm{~m}, 6 \mathrm{H})$; $4.30-4.28(\mathrm{~m}, 6 \mathrm{H}) .{ }^{13} \mathrm{C}$ NMR $\delta=142.19 ; 138.42 ; 133.68 ; 122.22$; $117.25 ; 97.80 ; 64.83 ; 64.50$.

\section{Electrochemical deposition}

Electrochemical depositions were performed in a glass cell installed with a platinum wire as the counter electrode. An Autolab PGstat12 potentiostat/galvanostat (EcoChemie) was employed as the power source. For copolymerization, mixed monomer solutions were prepared by varying the feed ratio of $\mathrm{EPh}$ in the EDOT-EPh mixture. The feed ratio was described as molar concentration of $\mathrm{EPh}$ in the total amount of monomer. For example, $0.1 \% \mathrm{EPh}$ feed ratio was prepared by mixing $\mathrm{EPh}-$ EDOT monomers at 1:999. For all the solutions, the concentration was kept at $0.02 \mathrm{M}$ in acetonitrile with $0.02 \mathrm{M}$ tetrabutyl ammonium perchlorate as counterion.

Pt microelectrode $\left(0.02 \mathrm{~cm}^{2}\right.$, Bioanalytical Systems, Inc, for EIS and CV tests) were used as the working electrode for the impedance and CV measurements of electrochemically deposited polymer films. For UV-vis-NIR spectroscopy studies, polymer films were electrochemically coated onto ITO glass slides $\left(4-8 \Omega \mathrm{sq}^{-1}\right.$, $0.5 \mathrm{~cm}^{2}$, Delta Technologies). Typically, $80 \mu \mathrm{A} \mathrm{cm}{ }^{-2}$ of constant current were employed for galvanostatic depositions. The reference was calibrated with $0.01 \mathrm{M}$ ferrocene in acetonitrile with $0.02 \mathrm{M}$ TBAP as supporting electrolyte and compared with $\mathrm{Ag} / \mathrm{AgCl}$ reference electrode (Fisher Scientific). The ferrocene $\mathrm{E}_{1 / 2}$ was measured as $0.11 \mathrm{~V}$ versus $\mathrm{Ag} / \mathrm{AgNO}_{3}$ and $0.48 \mathrm{~V}$ versus $\mathrm{Ag} / \mathrm{AgCl}$. Thus, for the potentials measured in organic solvents with $\mathrm{Ag} / \mathrm{AgNO} 3$ as a non-aqueous reference electrode, $+0.37 \mathrm{~V}$ was added into its value for better comparison with those measured in aqueous solutions against $\mathrm{Ag} / \mathrm{AgCl}$ reference electrode. The scan rate was $50 \mathrm{mV} \mathrm{s}^{-1}$.

\section{Characterization}

All the electrochemical impedance spectroscopy (EIS) and cyclic voltammetry (CV) characterizations of polymer films were performed on the same Autolab used for depositions. The tests were performed in $1 \times$ monomer-free phosphate buffered saline (PBS) solution with an $\mathrm{Ag} / \mathrm{AgCl}$ glass body electrode (Fisher Scientific) as the reference and a platinum foil as the counter electrode. The scan rate was $100 \mathrm{mV} \mathrm{s}^{-1}$ for CV. For EIS measurement, a $0.01 \mathrm{~V}$ bias versus $\mathrm{Ag} / \mathrm{AgCl}$ was applied. The frequency range scanned was from 1-100 kHz.

Optical and fluorescence microscopy was performed on a Nikon Eclipse LV100 POL microscope equipped with a Nikon Intensilight C-HGFI lamp. The images were collected via a Nikon DS-Ri1 camera.

UV-vis spectra were collected on a Shimadzu UV-3600 UV-visNIR spectrophotometer.

Scanning electron microscope images were collected on a Zeiss Auriga 60 CrossBeam focused ion beam (FIB)/scanning electron microscope (SEM). The acceleration voltage was $3 \mathrm{kV}$. A secondary electron detector was employed.

Mechanical measurements. The quantitative nanomechanical (QNM) modulus was calculated by PeakForce $\mathrm{QNM}^{\mathrm{TM}}$ AFM mode with Nanoscope Dimension 3100 software on a Bioscope Catalyst (Bruker Nano/Veeco) AFM. All samples were measured at room temperature in the dry state. A TAP150A probe (force constant of $5 \mathrm{~N} \mathrm{~m}^{-1}$ ) was used to indent the sample surface to a depth of about 1-2 $\mathrm{nm}$. Firstly, taking the adhesion between the tip and the surface into account, the reduced Young's modulus $E_{\mathrm{r}}$ was calculated from

$$
E_{\mathrm{r}}=\frac{3\left(F_{\mathrm{tip}}-F_{\mathrm{adh}}\right)}{4 \sqrt{R d^{3}}}
$$

where $F_{\text {tip }}$ and $F_{\text {adh }}$ are the, $R$ is the tip radius, $d$ is the deflection. The actual Young's modulus can be calculated from

$$
\frac{1}{E_{\mathrm{r}}}=\frac{\left(1-v_{\mathrm{s}}^{2}\right)}{E_{\mathrm{s}}}+\frac{\left(1-v_{\mathrm{I}}^{2}\right)}{E_{\mathrm{I}}}
$$

where $E_{\mathrm{s}}$ is the sample modulus, $E_{\mathrm{I}}$ is the indenter modulus, $v_{\mathrm{s}}$ and $v_{\mathrm{I}}$ are the Poisson ratio of the sample and the indenter, respectively. In our work $E_{I} \gg E_{\mathrm{s}}$, so we can neglect the second term of this equation. The force constant $K$ was calibrated by using thermal tuning with each measurement. To obtain an estimate of the probe radius $R, \mathrm{TiO}_{2}$ standards with well-defined curvature features were employed. By analysing the image generated from imaging the $\mathrm{TiO}_{2}$ standards, the accurate tip radius $R$ was obtained. Since the tip may wear out after scanning, the calibration was performed before each experiment. Three scans were taken at different spots for each sample. The modulus mapping was made by indenting $512 \times 512$ points over a $2 \mu \mathrm{m} \times 2 \mu \mathrm{m}$ image area. 
In vitro cell culture tests. All the polymer films were prepared by depositing onto gold-coated silicon substrates (approximately $0.2 \mathrm{~cm}^{2}$ ) at a charge density of $120 \mathrm{mC} \mathrm{cm}^{-2}$. For each group, 3 to 4 independent samples were prepared for each test. Prior to cell seeding, the samples were sterilized by immersing into ethanol for overnight. After rinsing with PBS 2 times, the samples were transferred into $50 \mu \mathrm{g} \mathrm{mg}^{-1}$ collagen I (from rat tail, Invitrogen) PBS solutions and incubated for $4 \mathrm{~h}$. After collagen coating, the samples were rinsed with PBS and transferred into a 24-well culture plate. Rat pheochromocytoma cells expressing green fluorescent protein (PC-12-Turbo-GFP, Marin Pharm) were seeded onto the samples at a density of $2 \times 104$ per well through a 22 gauge needle to dispense the cells. The cells were cultured in growth medium RPMI 1640 (Corning Cellgro) plus 5\% fetal calf serum (FCS), 10\% horse serum (HS), 2 mM L-alanyl-Lglutamine, $1 \%$ Pen-Strep and 1\% non essential amino acids (NEAA) overnight before switching to differentiation medium (RPMI 1640 with $150 \mathrm{ng} \mathrm{mL}{ }^{-1} \mathrm{NGF}, 1 \%$ FCS and 1\% HS). ${ }^{36}$ For cell viability tests, the cell numbers were calculated before switching to differentiation medium. For neurite length measurements, after day 4 , the differentiation media was replaced with freshly prepared new differentiation medium.

Cell viability tests. The cell culture medium was replaced with $0.25 \%$ trypsin solution (with $3.32 \mathrm{mM}$ EDTA, Corning) and incubated at $37{ }^{\circ} \mathrm{C}$ for 3 to 5 minutes to detach cells. The trypsin solution with cells was transferred to a $15 \mathrm{~mL}$ centrifuge tube. $5 \mathrm{~mL}$ fresh cell culture medium was added. The cell suspension was centrifuged at $200 \mathrm{rpm}$ for $5 \mathrm{~min}$ at room temperature. The medium was then pipetted out. $1 \mathrm{~mL}$ of new medium was added and the cell pellet was dispersed into the medium. $10 \mu \mathrm{L}$ of this cell suspension was withdrawn and mixed with $10 \mu \mathrm{L}$ trypan blue solution ( $0.4 \%$, Invitrogen), which only stains the dead cells. After mixing, the mixture was pipetted onto a cell counting chamber slide (Countess ${ }^{\mathrm{TM}}$, Invitrogen). The slide was placed into a Countess Automated Cell Counter (Invitrogen). The cell viability was given as the percentage of live cells in the total cell numbers. For each sample, 5 to 10 independent measurements were performed.

Neurite outgrowth measurements. The samples were transferred onto a glass slide that had PBS as the mounting medium after 4 days of exposure in differentiation medium. A cover glass was mounted onto the slide. The fluorescence images were collected under the Nikon Eclipse LV100 POL microscope. For each group, 3 to 4 samples were prepared and for each sample, 10 to 12 images on different spots of the film were taken. All the images were loaded into FIJI software. The length of neurites was measured by drawing a line from the neurite end to the junction between neurite cell bodies using FIJI software.

Statistics. The statistical analysis was performed with the computer program Minitab 17. The null hypothesis was that the cell viability for the different materials were all equal. The alternate hypothesis was at least one of the means of cell viability for the different materials were not equal. This was tested by performing four One-Way Analysis of Variance (ANOVA) tests: one ANOVA test for each day of data recording (day 2, day 5, day 8, day 11). The response of the ANOVA was cell viability and the factor was the material. In addition, a Dunnett method of multiple comparisons was performed with PEDOT as the control group level. All were performed at $95 \%$ confidence level $(\alpha=0.05)$.

\section{Results and discussion}

\section{Electrochemical polymerization of EDOT and EPh copolymers (PEDOT-co-EPh)}

EPh monomer was synthesized following Idzik et al.'s procedure. ${ }^{32}$ The oxidation potential of EPh and EDOT in acetonitrile was found to be at $+0.85 \mathrm{~V}$ and $+1.25 \mathrm{~V}$ versus SCE, respectively. It was suggested that the copolymer can be electrochemically synthesized by applying a voltage that was higher than both of the monomers in a mixed monomer solution. ${ }^{37}$ Fig. 2 shows cyclic voltammetry (CV) scans in the monomer solutions. Above $+1.2 \mathrm{~V}$ (vs. $\mathrm{Ag} / \mathrm{AgCl}$ ), EPh, EDOT and the monomer mixtures (1\% and $2 \%$ EPh feed ratio) were irreversibly oxidized. The integrated area under the CV curve expanded after each scan, confirming the formation of an electroactive film. Typically, the CV curves in EDOT solution during polymerization only showed a wide capacitive response as a result of overlapping redox peaks. Compared to EDOT, the CV sweeping in EPh solution showed a significantly decreased current response. Except for a redox peak at $+1.1 \mathrm{~V}$ that increase after each $\mathrm{CV}$ cycle, there was not much electroactivity in the EPh solution within the scan range. In the mixed monomer solutions, although the $\mathrm{CV}$ curves showed similar wide capacitance that was contributed by the EDOT moieties there was an overall decrease of current response after each CV cycle, especially in the negative potential range compared to that in neat EDOT solution. This decreased electroactivity suggested that the less electroactive EPh, even at relatively low concentration (2\%), could be simultaneously oxidized with EDOT and incorporated into the film, as evident from the CV curve.
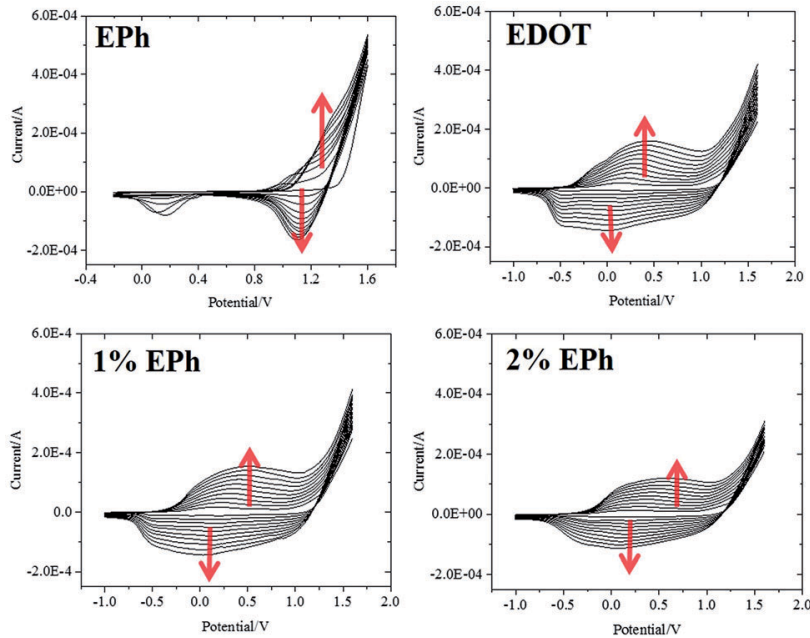

Fig. 2 First 10 cycles of CV recorded during potentiodynamic polymerizations in monomer solutions (versus $\mathrm{Ag} / \mathrm{AgCl}$ ). Red arrows indicate expanded CV curves after each cycle. 


\section{Optical properties of PEDOT-co-EPh}

PEDOT-co-EPh copolymer films were obtained by applying an oxidizing current $\left(80 \mu \mathrm{A} \mathrm{cm}{ }^{-2}\right.$, potential recorded around $+2.4 \mathrm{~V}$ ) in a series of mixed monomer solutions. The galvanostatic deposition method was chosen as it gives better control of the film deposition. It was found that the film color changed systemically as the amount of EPh in the solution increased. As shown in Fig. 3, the electrochemically deposited neat PEDOT had a characteristic sky-blue color due to its partially oxidized state. The film deposited at $0.1 \% \mathrm{EPh}$ showed a slightly yellowish color. The yellow tone became much clearer at $0.2 \% \mathrm{EPh}$ while at $1 \%$ the film turned into a golden color. Further increasing the EPh feed ratio led to a reddish color in the resultant films. At 20\%, the golden color became pinkish. The homopolymer PEPh had a deep red color. The UV-vis-NIR spectra of the galvanostatically deposited PEDOT-co-EPh polymer films, formed with the same total deposition charge (72 $\mathrm{mC} \mathrm{cm} \mathrm{cm}^{-2}$ ) showed that the copolymerization shifted the absorption maximum to shorter wavelengths. As shown in Fig. 4, the neat PEDOT films had a low and broad absorption from visible light to near IR. Adding only $0.1 \% \mathrm{EPh}$ did not significantly change the absorption properties. However, at $0.2 \% \mathrm{EPh}$, broad peaks from 500 and $760 \mathrm{~nm}$ started to emerge in the spectra. This explains the yellowish tone on the overall blue film. At $0.5 \% \mathrm{EPh}$, a new absorption peak at $\sim 650 \mathrm{~nm}$ was found, accompanied by a shoulder around $500 \mathrm{~nm}$. It also had a high absorption from 900 to $1400 \mathrm{~nm}$, peaking around $1100 \mathrm{~nm}$. Increasing the $\mathrm{EPh}$ feed ratio to $1 \%$, the absorption at $500 \mathrm{~nm}$ increased. A decrease of absorption at longer wavelengths was also found.

At higher EPh feed ratios, there was a further blue-shift of wavelength of the absorption maxima. Additional peaks around $450 \mathrm{~nm}$ also started to appear. For example, $10 \% \mathrm{EPh}$ feed ratio resulted in twin peaks at $432 \mathrm{~nm}$ and $500 \mathrm{~nm}$ in the spectrum. The absorption around $450 \mathrm{~nm}$ increased with further $\mathrm{EPh}$ concentration in the feed. At $20 \%$, the relative height of $\sim 450 \mathrm{~nm}$ peak to its $500 \mathrm{~nm}$ counterpart greatly increased. For the PEPh homopolymer films, the $500 \mathrm{~nm}$ absorption became a shoulder peak.

The $\lambda_{\text {max }}$ of the films deposited in different EPh feed ratio is summarized in Fig. 4 b. It was clear that $\lambda_{\max }$ decreased quickly at very low EPh feed ratios. The absorption maximum blue shifted from over $1000 \mathrm{~nm}$ for $0.1 \% \mathrm{EPh}$ to around $500 \mathrm{~nm}$ for $1 \% \mathrm{EPh}$. The absorption at longer wavelength (900 to $1600 \mathrm{~nm}$ ) also decreased quickly at less than $1 \% \mathrm{EPh}$, while the absorption at shorter wavelengths (400 to $600 \mathrm{~nm}$ ) increased with increasing EPh feed (Fig. 4c). It was thus possible to tune the absorption maximum of the films by tuning the EPh feed ratio from $0.1 \%$ to $1 \%$.

As is usual with electrochemical deposition, the film thickness could be readily controlled with the deposition charge. At higher deposition charges, thicker films were formed, as is evident from the increased optical absorption. Fig. 5 show the UV-vis-NIR spectra of the PEDOT and PEPh as a function of deposition charge. The broad absorption of PEDOT starting from the visible to IR range generally increased with increasing deposition charge. The characteristic $\lambda_{\text {max }}$ of PEPh was found to be between $400 \mathrm{~nm}$ and $600 \mathrm{~nm}$. An absorption around $430 \mathrm{~nm}$ increased with deposition charge. At low deposition charge $\left(0.4 \mathrm{mC} \mathrm{cm}^{-2}\right)$, a broad absorption peak of PEPh homopolymer appeared around 500-600 nm. With increasing deposition charge, two peaks, around 450 and 550 respectively, could be identified. The peak around 450 increased with the increasing deposition charge. It became the main $\lambda_{\max }$ at higher deposition charges while the $\sim 550$ peak became a shoulder peak.

\section{Surface morphology of PEDOT-co-EPh films}

The copolymerization significantly changed the surface morphology of PEDOT films. As shown in Fig. 6, when using perchlorate as dopant, the PEDOT film had a fuzzy, fibrous surface composed
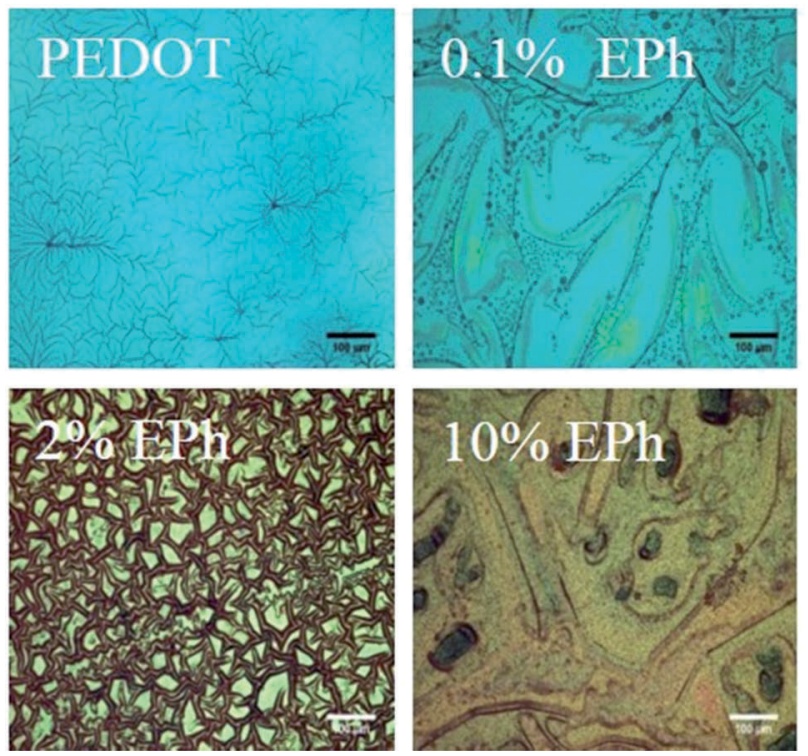
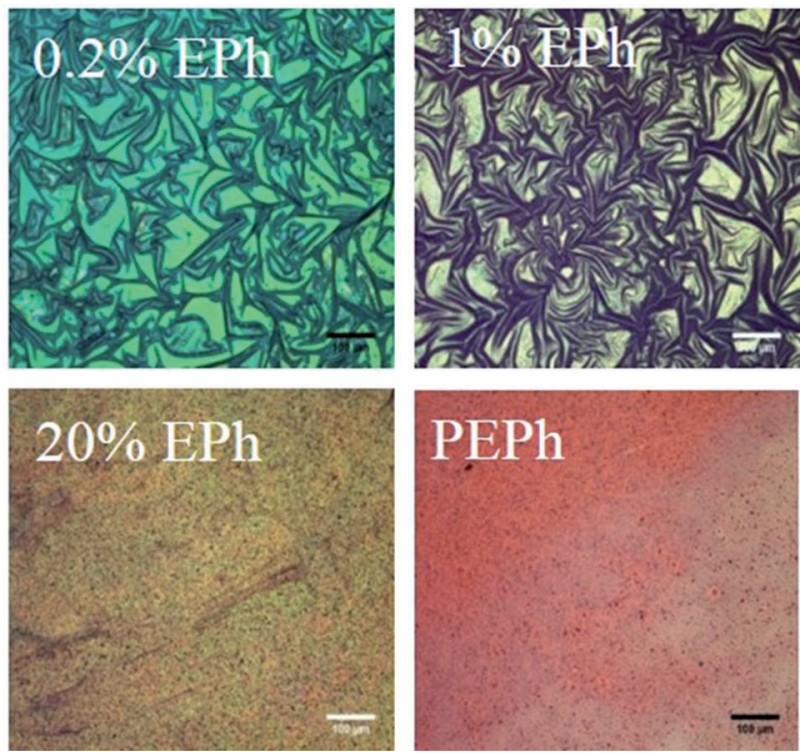

Fig. 3 Optical micrographs of P(EDOT-CO-EPh) films (transmitted light). Scale bars represent $100 \mu \mathrm{m}$. 
a

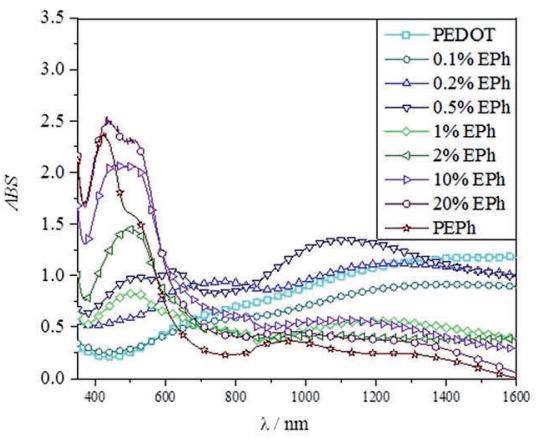

b

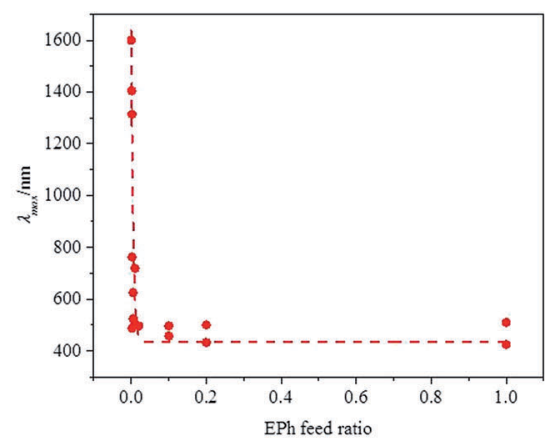

c

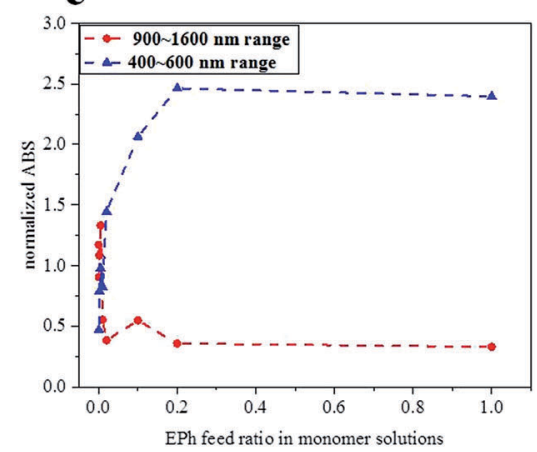

Fig. 4 (a) UV-vis-NIR absorption of the PEDOT-co-EPh copolymers; (b) $\lambda_{\max }$ versus composition; (c) normalized absorption in near-infrared $(900-1600 \mathrm{~nm})$ and visible $(400-600 \mathrm{~nm})$ wavelength range versus composition.
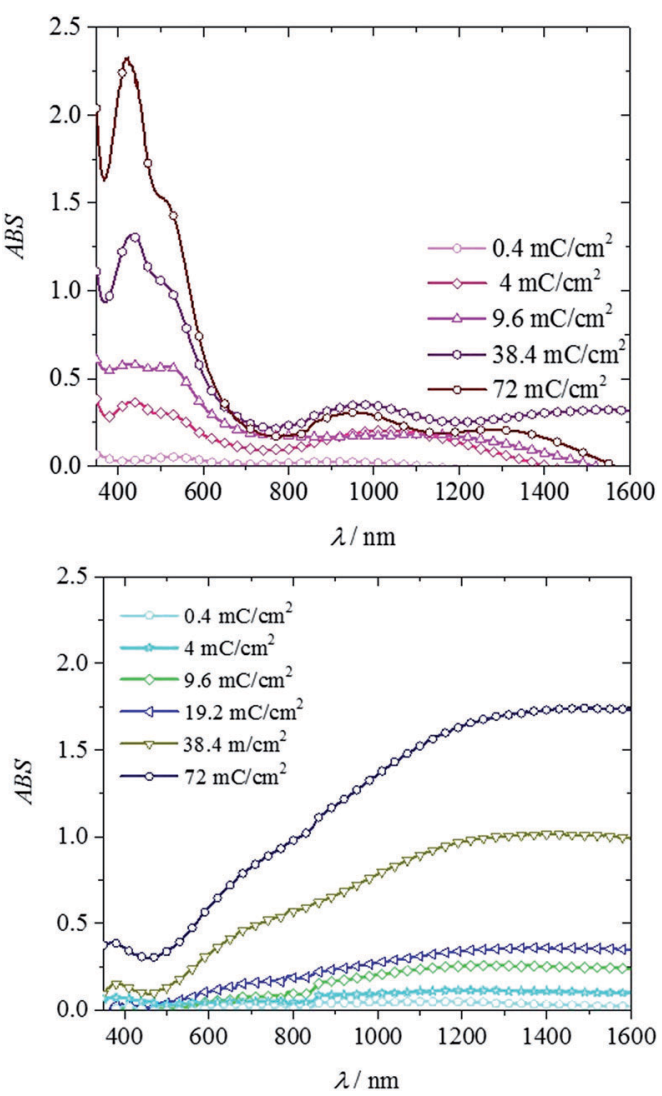

Fig. 5 UV-vis-NIR spectra of polymers at different deposition charges. Top: PEPh; bottom: PEDOT.

of $\sim 100 \mathrm{~nm}$ polymer islands interconnected by numerous nanofibers. It was found that only $0.1 \% \mathrm{EPh}$ in the monomer feed significantly changed the surface morphology. The nanofibers completely disappeared from the surface. Instead, the polymer islands packed together and fused onto the underlying layer. At $1 \% \mathrm{EPh}$, the texture of film became very smooth. No fine structures could be found on the surface except for sparsely distributed $\sim 200 \mathrm{~nm}$ particles. At $10 \% \mathrm{EPh}$ in the feed, the film started to demonstrate a bumpy morphology. At $20 \%$, the surface was composed of $\sim 500 \mathrm{~nm}$ large bumps which in turn were
A
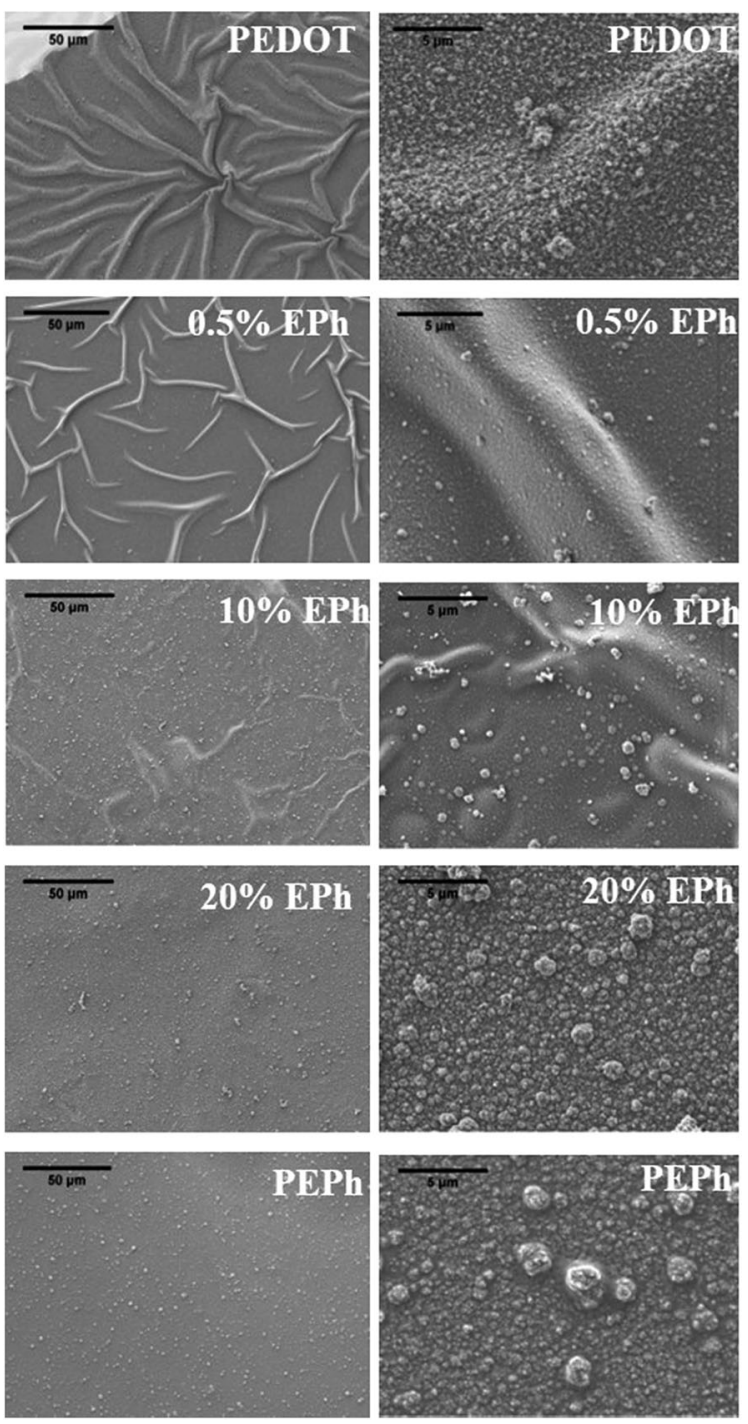

Fig. 6 SEM images of P(EDOT-CO-EPh) films as a function of EPh feed ratio; (A) column: lower magnification (scale bar represents $50 \mu \mathrm{m}$ ); (B) column: higher magnification (scale bar represents $5 \mu \mathrm{m}$ ). 
formed by clusters of $\sim 50 \mathrm{~nm}$ small particles. This morphology was similar to the surface morphology of the PEPh homopolymer.

Another observation was the buckling of the polymer surface. The polymer films were all deposited onto ITO substrates from acetonitrile solutions and then dried in air before being subjected to morphological observations in the vacuum of the SEM. Acetonitrile is known to cause modest swelling of PEDOT. ${ }^{38}$ On a substrate that has no specific interactions with the film, the swelling and drying can lead to film buckling. The buckling pattern was thus a function of the extension of swelling (the interactions between the solvent molecules) and the mechanical properties of the film itself. For PEDOT, dense buckling patterns were formed on the surface. The ridges in the buckled film were about $5 \mu \mathrm{m}$ wide. It was clear that copolymerizing with EPh substantially decreased the extent of buckling seen in the SEM. From $0.1 \%$ to $10 \% \mathrm{EPh}$ feed ratio, the buckling was significantly reduced. At $20 \% \mathrm{EPh}$ and above the films remained smooth and flat, with no buckling. This transition is evidently related to the increase in mechanical properties and decrease in solvent swelling due to crosslinking.

\section{Increased impedance of PEDOT-co-EPh}

The charge transport properties of the PEDOT-co-EPh polymer films were characterized by electrochemical impedance spectroscopy (EIS). With its electronic and ionic conductivity and large effective surface area, PEDOT coatings have been shown to greatly decrease the system impedance of metal electrodes. As shown in Fig. 7, at frequencies below $1 \mathrm{kHz}$, a decrease of $\sim 2$ orders of magnitude could be found with PEDOT coatings.

The EPh-crosslinked PEDOT, however, became much less conducting than the PEDOT homopolymer. With only $0.1 \%$ EPh monomer in the feed, the copolymer film showed similar impedance spectra as the PEDOT film at the same deposition charge density. By increasing the $\mathrm{EPh}$ feed ratio to $0.2 \%$, a slight increase in impedance magnitudes was found. With this conducting polymer coating, the impedances at lower frequencies $(1 \mathrm{~Hz}$ to $100 \mathrm{~Hz}$ ) were still much lower than the bare electrode. However, it showed slightly higher impedances than the bare electrode at higher frequencies.

When the EPh feed ratio was increased to 1\%, the impedance benefit from the conducting polymer coating was diminished. At $1 \% \mathrm{EPh}$ feed ratio, the impedance magnitude of the coated electrode was similar to that of the bare electrode. A slight increase of impedance was found at higher frequency ranges (from $1 \mathrm{kHz}$ to $100 \mathrm{kHz}$ ). With further increases in the EPh feed ratio, the copolymer film became even more insulating. Above $2 \%$, the impedance of the coating was higher than that of the bare Pt. Similar insulating effects were found for 10\%, 20\% and 50\% EPh films (Fig. 7).

Fig. $7 \mathrm{~b}$ shows the impedance magnitudes at $1 \mathrm{~Hz}$ and $1 \mathrm{kHz}$ for different $\mathrm{EPh}$ feed ratios. The initially low impedance increased substantially with increasing $\mathrm{EPh}$ feed ratio, becoming higher than the bare electrode for EPh concentrations above $1 \%$. The maximum was found at $10 \%$ and then plateaued for higher EPh feed ratios.
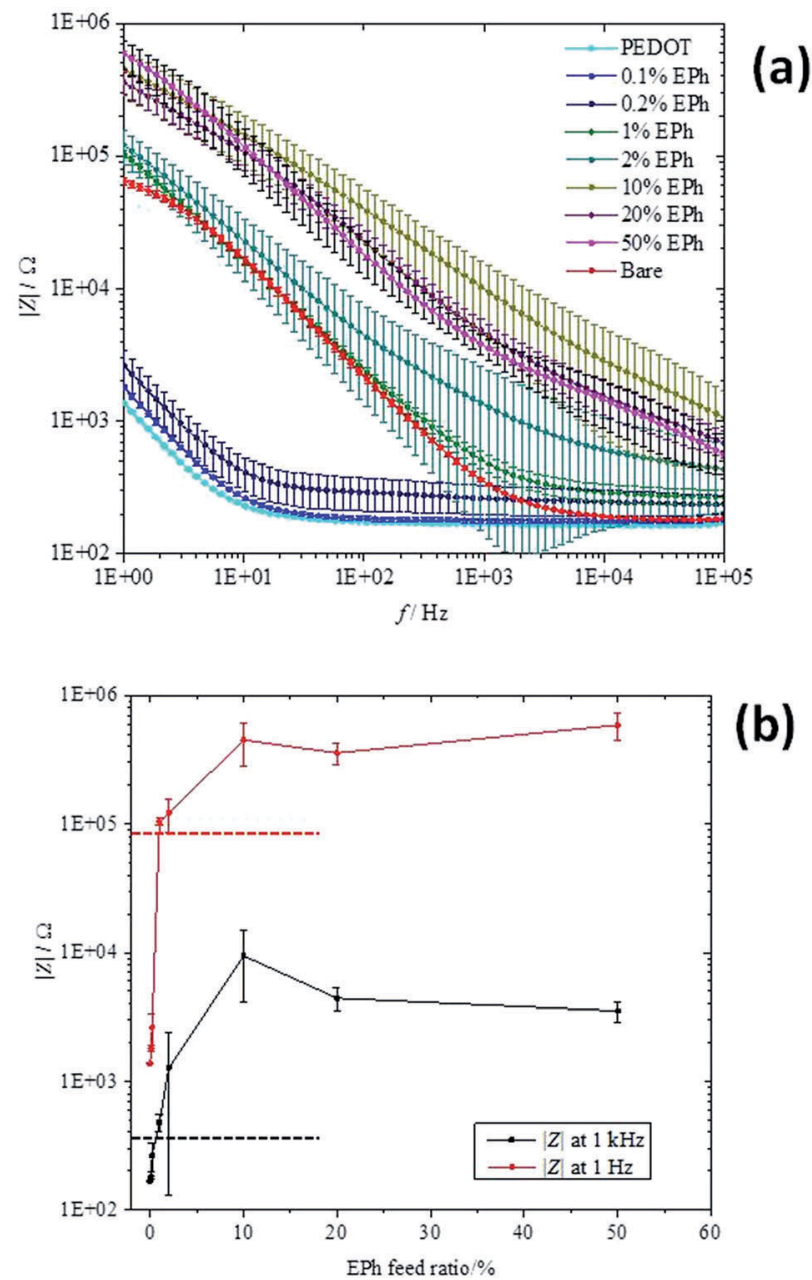

Fig. 7 (a) Impedance amplitude of PEDOT and PEDOT-CO-EPh copolymers as a function of frequency; (b) impedance amplitude at $1 \mathrm{~Hz}$ and $1 \mathrm{kHz}$ for the PEDOT-CO-EPh copolymers as a function of EPh content; dashed lines indicate the impedance amplitude of bare Pt electrode at $1 \mathrm{~Hz}$ (red) and $1 \mathrm{kHz}$ (black), respectively (deposition charge: $288 \mathrm{mC} \mathrm{cm}^{-2}$ ).

For the purpose of neural recording and stimulations, it is desirable to maintain low impedance at the electrode/tissue interface. Previously, it was found that the low impedance of conducting polymer coated electrodes could be correlated with the high effective surface areas of its fuzzy morphology. ${ }^{39}$ Here two factors could be related to the decreased conductivity of the polymer coatings. From the morphology, it was likely that the relatively dense surface from crosslinking would not support sufficient ion transport through the interface, resulting in higher impedance. On the other hand, from the UV-vis-NIR absorption, it was found that the copolymerization decreased the effective conjugation length of the polymer film. It was thus possible that the EPh unit served as a defect to the conjugated backbone. ${ }^{40,41}$ With shorter conjugation chain length, the material became less conducting.

\section{Mechanical properties}

The addition of EPh clearly improved the mechanical cohesion of the PEDOT films. As discussed before, the copolymerization 
had shown effects on the buckling behaviour of the electrochemically deposited films. Macroscopically, much more uniform and robust films were observed with higher EPh feed ratios. We found that above $10 \% \mathrm{EPh}$ feed, the thicker copolymer films tended to easily detach from ITO substrate as an intact whole. However, without any supporting matrix, these films were still too brittle for tensile tests.

In order to evaluate the effects of crosslinking on the mechanical properties of PEDOT films, a PeakForce QNM $^{\mathrm{TM}}$ AFM method was adopted. The films were indented at a nanometer depth (1-2 nm) with an AFM tip. The typical force constant of the tip was $5 \mathrm{~N} \mathrm{~m}^{-1}$ with a frequency of $150 \mathrm{kHz}$. The tip radius ranged from $2-12 \mathrm{~nm}$ depending on manufacturing conditions. The precise tip radius was calibrated with a $\mathrm{TiO}_{2}$ standard before each set of measurements. The elastic modulus of the materials was calculated from the force curves using the Derjaguin-Muller-Toporov (DMT) model. $^{42-44}$ Fig. 8 shows the topological map and modulus histogram of PEDOT, $0.1 \%$ and $0.5 \% \mathrm{EPh}$ feed ratios. The latter two compositions were compared with PEDOT since they maintained the relatively high conductivity needed for neural probe coatings. It was found that the indentation modulus calculated from $\mathrm{QNM}^{\mathrm{TM}}$ mode with the DMT model for PEDOT was $0.75 \pm 0.16 \mathrm{GPa}$. The film deposited at $0.1 \% \mathrm{EPh}$ had a modulus of $1.35 \pm 0.48 \mathrm{GPa}$. However, at $0.5 \% \mathrm{EPh}$, the modulus became $4.9 \pm 2.1 \mathrm{GPa}$, which was $\sim 5$ times larger than the PEDOT film.

It should be noted that mechanical properties of neat PEDOT films are known to be influenced by factors such as dopant and humidity. Green et al. measured the indentation modulus of PEDOT films with different small molecule dopants. ${ }^{16}$ It was found that larger dopants lead to higher modulus. The indentation modulus for electrochemically polymerized PEDOT film doped with perchlorate was measured as $1.29 \pm 0.72 \mathrm{GPa}^{16}$

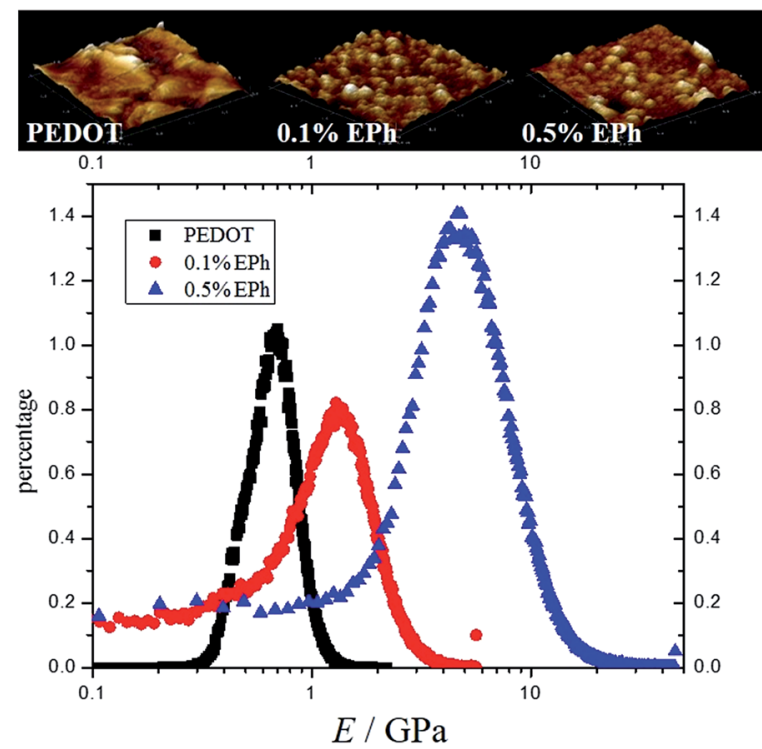

Fig. 8 AFM QNM modulus distribution. (a) AFM image of the polymer films ( $2 \mu \mathrm{m}$ by $2 \mu \mathrm{m}$ ); (b) AFM QNM modulus distribution of PEDOT, $0.1 \%$ EPh and $0.5 \%$ EPh
However, using a similar AFM PeakForce method, the same group reported that the average modulus of perchlorate doped PEDOT (PEDOT:ClO 4 ) film was $68.0 \mathrm{MPa} .{ }^{45}$ The authors suggested that the indentation method suffered from the large tip radius $(100 \mu \mathrm{m})$, which would likely have contributed to the overestimation of the stiffness. ${ }^{45}$ However, in this research, the modulus measured from 2-20 nm tip nanoindentation was also found to be in the GPa range. One possible factor for this discrepancy is the different calibration methods employed between the two AFM PeakForce measurements. In Hassarati et al.'s paper, ${ }^{45}$ the calibration of tips was performed on a relatively soft PDMS standard (with a modulus around $1 \mathrm{MPa}$ ). The relative calibration method forces the value of ratio $K / R^{0.5}$ to be correct at a given indentation depth. This assumes limited modulus difference between standards and the samples to be measured. As the modulus of PEDOT was expected to be around hundreds of MPa to several GPa range, we found that the modulus varies dramatically between groups when using this soft standard for calibration. In order to measure this large range of modulus in a consistent manner, a universal calibration method was adopted in this research. The force constant of the cantilever was calibrated through thermal tuning and the precise tip radius was measured through analysing standard $\mathrm{TiO}_{2}$ samples.

The relative humidity $(\mathrm{rH})$ can also have a significant influence on the stiffness of the PEDOT films. Lang et al. ${ }^{46}$ found that for cast PEDOT:PSS (Baytron P) films, the Young's modulus varied from $0.9 \pm 0.2 \mathrm{GPa}$ at $55 \% \mathrm{rH}$ to $2.8 \pm 0.5 \mathrm{GPa}$ at $23 \% \mathrm{rH}$. However it should be noted that in this PEDOT:PSS dispersion, excessive PSS was required to stabilize the electrolyte complex. ${ }^{47}$ Typically, the weight ratio for PSS to PEDOT can vary from $2.5: 1$ to $6: 1$. Also, PSS is highly hygroscopic. The dependence of the Young's modulus on humidity can thus be largely attributed to the hydration kinetics of PSS. ${ }^{48}$ The counterion concentration in these electrochemically polymerized PEDOT films, on the other hand, is substantially lower. ${ }^{49}$ Thus, variation of stiffness with humidity is expected to be smaller. It was found that the modulus of hydrated PEDOT: $\mathrm{ClO}_{4}$ films decreased for $38 \%$ while PEDOT:PSS over $90 \%{ }^{45}$

\section{Biocompatibility}

The excellent biocompatibility of PEDOT has been well documented. ${ }^{25,50}$ However, it was unknown if the changes in chemical composition of PEDOT- $c o$-EPh copolymers as well as the surface stiffness would change the biocompatibility of the films. For this purpose, PC-12 cell lines were employed as an in vitro model to test the biocompatibility of PEDOT-co-EPh films with different $\mathrm{EPh}$ compositions. The cell viability on day 2, 5, 8 and 11 after seeding was tested. It was found that all of the samples well supported the cell growth. As shown in Fig. 9a, the viability across all days remained above $80 \%$. A slight drop of viability on day 8 for $0.2 \%$ EPh feed ratio $(p<0.05)$ and on day 11 for $1 \% \mathrm{EPh}(p<0.001)$ was found, but it still remained above $70 \%$.

After switching to differentiation medium, the outgrowth of neurites was measured. As shown in Fig. 9b, it was found that the PC12 cells grew well on these substrates, with a large percentage of cells expressing interconnected neurites. The mean neurite length 


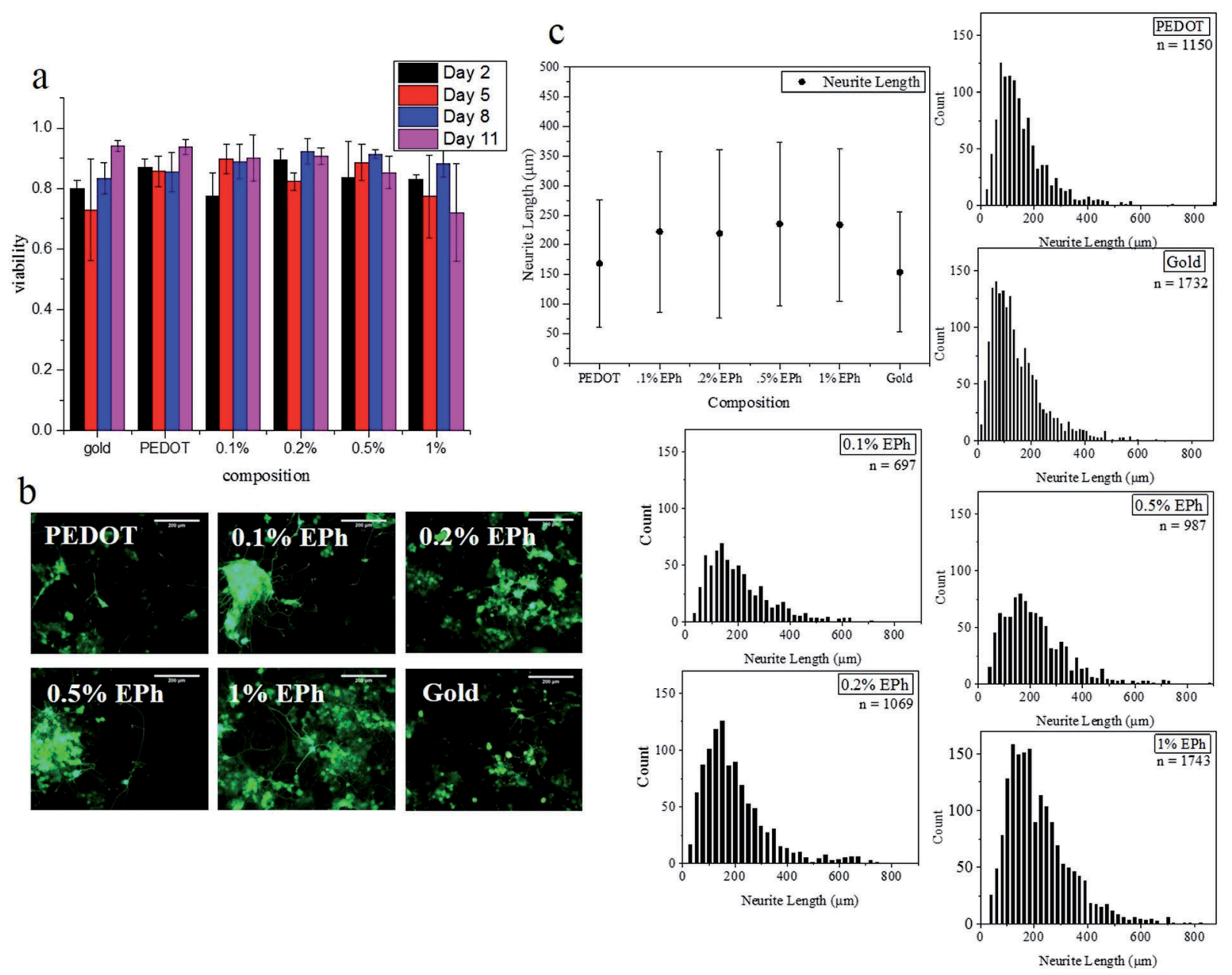

Fig. 9 Influence of PEDOT-CO-EPh composition on PC-12 viability and neurite outgrowth in vitro. (a) PC-12 cell viability cultured on gold and various polymer-coated substrates on days 2, 5, 8 and 11. (b) Representative fluorescence images of differentiated cells. Scale bars indicate $200 \mu \mathrm{m}$ (c) mean neurite lengths of the PC-12 cells cultured on polymer films and corresponding neurite length distribution.

was found to be between 150 to $250 \mu \mathrm{m}$. Fig. 9c shows the neurite distributions of the cells cultured on different polymer substrates. Most neurites were around 100-200 $\mu \mathrm{m}$. However, neurites that grew over $800 \mu \mathrm{m}$ were also observed. These results demonstrate that the EPh crosslinked PEDOT showed no significant issues with cytotoxicity toward PC-12 cells. With the ability to deliver charge, these polymer substrates could also potentially be employed to guide cell growth through electrical stimulation. ${ }^{51}$

\section{Conclusions}

A three-armed EDOT crosslinker with a benzene core, EPh, was electrochemically copolymerized with EDOT in mixed monomer solutions to create heavily branched or crosslinked PEDOT-co-EPh copolymer coatings on metal electrodes. It was found that copolymerization with EPh dramatically changed the optical absorption of the PEDOT-co-EPh films. The absorption at longer wavelengths (900 to $1600 \mathrm{~nm}$ ) decreased quickly with increasing EPh feed ratio, while new absorptions between 400 to $600 \mathrm{~nm}$ increased at the same time. As a result, the film color gradually changed from sky blue for PEDOT to pinkish and red for PEPh. The crosslinking also led to a denser surface morphology of the resultant films, as seen in SEM. Electrochemical impedance spectroscopy showed that the copolymerization had a negative effect on the impedance of the coatings. At the lowest EPh feeds (less than 0.02\%), the PEDOT-co-EPh films had comparable impedances to neat PEDOT coatings. However, the impedance quickly increased with higher $\mathrm{EPh}$ feed ratios. Macroscopically, more coherent film formation was observed at higher $\mathrm{EPh}$ feed ratios, suggesting improved mechanical strength. This observation was confirmed by AFM nanoindentation tests, where at $0.5 \% \mathrm{EPh}$, the film had $5 \times$ larger $\mathrm{QNM}^{\mathrm{TM}}$ indentation modulus than PEDOT. Lastly, in vitro cell culture tests showed that the PEDOT-co-EPh films exhibited excellent biocompatibility to PC-12 cell lines, with limited cytotoxicity and the ability to support neurite outgrowth. Our results suggest that for neural interface applications, the materials expected to be of most interest will be PEDOT-co-EPh copolymers with less than $1 \% \mathrm{EPh}$. These materials retain the ability to facilitate charge transport between the electrode and tissue, yet also have substantially improved mechanical properties. 


\section{Acknowledgements}

This research was supported in part by the National Institutes of Health through EUREKA grant 1RO1EB010892, by the Defense Advanced Research Projects Agency (DARPA) grant N66001-11-C-4190, and by the University of Delaware.

\section{Notes and references}

1 C. Schmidt, V. Shastri, J. Vacanti and R. Langer, Stimulation of neurite outgrowth using an electrically, Proc. Natl. Acad. Sci. U. S. A., 1997, 94, 8948-8953.

2 N. Gomez and C. E. Schmidt, Nerve growth factor-immobilized polypyrrole: bioactive electrically conducting polymer for enhanced neurite extension, J. Biomed. Mater. Res., Part A, 2006, 81, 135-149.

3 W.-K. Oh, O. S. Kwon and J. Jang, Conducting Polymer Nanomaterials for Biomedical Applications: Cellular Interfacing and Biosensing, Polym. Rev., 2013, 53, 407-442.

4 D.-H. Kim, J. a. Wiler, D. J. Anderson, D. R. Kipke and D. C. Martin, Conducting polymers on hydrogel-coated neural electrode provide sensitive neural recordings in auditory cortex, Acta Biomater., 2010, 6, 57-62.

5 M. Gerard, A. Chaubey and B. D. Malhotra, Application of conducting polymers to biosensors, Biosens. Bioelectron., 2002, 17, 345-359.

6 D.-H. Kim, et al., in Indwelling Neural Implants: Strategies for Contending with the In-Vivo Environment, ed. W. M. Reichert, Taylor and Francis, 2007.

7 P. Fattahi, G. Yang, G. Kim and M. R. Abidian, A review of organic and inorganic biomaterials for neural interfaces, Adv. Mater., 2014, 26, 1846-1885.

8 T. D. Y. Kozai, et al., Nanotechnology and Neuroscience: Nanoelectronic, Photonic and Mechanical Neuronal Interfacing, Springer, New York, 2014, pp. 71-131, DOI: 10.1007/978-14899-8038-0.

9 C. Saltó, et al., Control of neural stem cell adhesion and density by an electronic polymer surface switch, Langmuir, 2008, 24, 14133-14138.

10 K. a. Ludwig, et al., Poly(3,4-ethylenedioxythiophene) (PEDOT) polymer coatings facilitate smaller neural recording electrodes, J. Neural Eng., 2011, 8, 014001.

11 X. Cui and D. C. Martin, Electrochemical deposition and characterization of poly(3,4-ethylenedioxythiophene) on neural microelectrode arrays, Sens. Actuators, B, 2003, 89, 92-102.

12 X. Cui and D. C. Martin, Fuzzy gold electrodes for lowering impedance and improving adhesion with electrodeposited conducting polymer ${ }^{\circledR}$ lms, Sens. Actuators, A, 2003, 103, 384-394.

13 S. J. Wilks, S. M. Richardson-burns, J. L. Hendricks, D. C. Martin and K. J. Otto, Poly(3,4-ethylenedioxythiophene) as a microneural interface material for electrostimulation, Front. Neuroeng., 2009, 2, 1-8.

14 D. C. Martin, et al., The Morphology of Poly(3,4-Ethylenedioxythiophene), Polym. Rev., 2010, 50, 340-384.

15 P. J. Molino, et al., Influence of Biodopants on PEDOT Biomaterial Polymers: Using QCM-D to Characterize
Polymer Interactions with Proteins and Living Cells, Adv. Mater. Interfaces, 2014, 1, 1300122.

16 S. Baek, R. a. Green and L. Poole-Warren, Effects of dopants on the biomechanical properties of conducting polymer films on platinum electrodes, J. Biomed. Mater. Res., Part A, 2014, 102, 2743-2754.

17 D. Khodagholy, et al., Highly conformable conducting polymer electrodes for in vivo recordings, Adv. Mater., 2011, 23, $\mathrm{H} 268-\mathrm{H} 272$.

18 J. K. Nguyen, et al., Mechanically-compliant intracortical implants reduce the neuroinflammatory response, J. Neural Eng., 2014, 11, 056014.

19 J. P. Harris, et al., Mechanically adaptive intracortical implants improve the proximity of neuronal cell bodies, J. Neural Eng., 2011, 8, 066011.

20 K. a. Potter, A. C. Buck, W. K. Self and J. R. Capadona, Stab injury and device implantation within the brain results in inversely multiphasic neuroinflammatory and neurodegenerative responses, J. Neural Eng., 2012, 9, 046020.

21 L. Ouyang, C. L. Shaw, C.-C. Kuo, A. L. Griffin and D. C. Martin, In vivo polymerization of poly(3,4-ethylenedioxythiophene) in the living rat hippocampus does not cause a significant loss of performance in a delayed alternation task, J. Neural Eng., 2014, 11, 026005.

22 X. T. Cui and D. D. Zhou, Poly(3,4-Ethylenedioxythiophene) for Chronic Neural Stimulation, IEEE Trans. Neural Syst. Rehabil. Eng., 2007, 15, 502-508.

23 R. Green, et al., Electrochemical stability of poly(ethylene dioxythiophene) electrodes, in The 5th IEEE/EMBS conference on Neural Engineering, 2011, vol. 5, pp. 566-569.

24 T. A. Kung, et al., Regenerative Peripheral Nerve Interface Viability and Signal Transduction with an Implanted Electrode, Plast. Reconstr. Surg., 2014, 133, 1380-1394.

25 M. Asplund, et al., Toxicity evaluation of PEDOT/biomolecular composites intended for neural communication electrodes, Biomed. Mater., 2009, 4, 045009.

26 C. Teng, X. Lu, Y. Zhu, M. Wan and L. Jiang, Polymer in situ embedding for highly flexible, stretchable and water stable PEDOT:PSS composite conductors, RSC Adv., 2013, 3, 7219.

27 C. Chen, et al., Mechanical characterizations of cast poly(3,4-ethylenedioxythiophene):poly(styrenesulfonate)/polyvinyl alcohol thin films, Synth. Met., 2011, 161, 2259-2267.

28 S. Ghosh and O. Inganäs, Conducting Polymer Hydrogels as 3D Electrodes: Applications for Supercapacitors, Adv. Mater., 1999, 11, 1214-1218.

29 S. Ghosh and O. Inganäs, Electrochemical Characterization of Poly(3,4-ethylene dioxythiophene) Based Conducting Hydrogel Networks, J. Electrochem. Soc., 2000, 147, 1872-1877.

30 I. F. Perepichka, et al., Hydrophilic Oligo (oxyethylene)Derivatized Optoelectroelectrochemical Properties and Solid-State Chromism, Chem. Mater., 2002, 14, 449-457.

31 K. R. Idzik, et al., Electrochemical and spectral properties of meta-linked 1,3,5-tris(aryl)benzenes and 2,4,6-tris(aryl)-1phenoles, and their polymers, Electrochim. Acta, 2010, 55, 7419-7426. 
32 K. R. Idzik, R. Beckert, S. Golba, P. Ledwon and M. Lapkowski, Synthesis by Stille cross-coupling procedure and electrochemical properties of C3-symmetric oligoarylobenzenes, Tetrahedron Lett., 2010, 51, 2396-2399.

33 J. Roncali, P. Leriche and a. Cravino, From One- to ThreeDimensional Organic Semiconductors: In Search of the Organic Silicon?, Adv. Mater., 2007, 19, 2045-2060.

34 A. I. Cooper, Conjugated Microporous Polymers, Adv. Mater., 2009, 21, 1291-1295.

35 S. S. Zhu and T. M. Swager, Conducting Polymetallorotaxanes: Metal Ion Mediated Enhancements in Conductivity and Charge Localization, J. Am. Chem. Soc., 1997, 119, 12568-12577.

36 D. G. Drubin, S. C. Feinstein, E. M. Shooter and M. W. Kirschner, Nerve growth factor-induced neurite outgrowth in PC12 cells involves the coordinate induction of microtubule assembly and assembly-promoting factors, J. Cell Biol., 1985, 101, 1799-1807.

37 T. Yohannes, J. C. Carlberg, O. Inganäs and T. Solomon, Electrochemical and spectroscopic characteristics of copolymers electrochemically synthesized from 3-methylthiophene and 3,4-ethylenedioxy thiophene, Synth. Met., 1997, 88, 15-21.

38 G. Zotti, G. Schiavon and S. Zecchin, A Polyconjugated Polymer with a High Spin Density Polaron State, Chem. Mater., 1999, 11, 3624-3628.

39 X. Cui, J. F. Hetke, J. a. Wiler, D. J. Anderson and D. C. Martin, Electrochemical deposition and characterization of conducting polymer polypyrrole/PSS on multichannel neural probes, Sens. Actuators, A, 2001, 93, 8-18.

$40 \mathrm{H}$. Ho, et al., Colorimetric and Fluorometric Detection of Nucleic Acids Using Cationic Polythiophene Derivatives, Angew. Chem., Int. Ed., 2002, 41, 1548-1551.

41 M. Leclerc, Optical and Electrochemical Transducers Based on Functionalized Conjugated Polymers, Adv. Mater., 1999, 11, 1491-1498.
42 K. Sweers, K. van der Werf, M. Bennink and V. Subramaniam, Nanomechanical properties of $\alpha$-synuclein amyloid fibrils: a comparative study by nanoindentation, harmonic force microscopy, and Peakforce QNM, Nanoscale Res. Lett., 2011, 6,270 .

43 M. E. Dokukin and I. Sokolov, Quantitative mapping of the elastic modulus of soft materials with HarmoniX and PeakForce QNM AFM modes, Langmuir, 2012, 28, 16060-16071.

44 J. Landoulsi and V. Dupres, Direct AFM force mapping of surface nanoscale organization and protein adsorption on an aluminum substrate, Phys. Chem. Chem. Phys., 2013, 15, 8429-8440.

45 R. T. Hassarati, et al., Stiffness quantification of conductive polymers for bioelectrodes, J. Polym. Sci., Part B: Polym. Phys., 2014, 52, 666-675.

46 U. Lang, N. Naujoks and J. Dual, Mechanical characterization of PEDOT:PSS thin films, Synth. Met., 2009, 159, 473-479.

47 M. M. De Kok, et al., Modification of PEDOT:PSS as hole injection layer in polymer LEDs, Phys. Status Solidi A, 2004, 201, 1342-1359.

48 S. R. Dupont, F. Novoa, E. Voroshazi and R. H. Dauskardt, Decohesion Kinetics of PEDOT:PSS Conducting Polymer Films, Adv. Funct. Mater., 2014, 24, 1325-1332.

49 G. Zotti, et al., Electrochemical and XPS Studies toward the Role of Monomeric and Polymeric Sulfonate Counterions in the Synthesis, Composition, and Properties of Poly(3,4-ethylenedioxythiophene), Macromolecules, 2003, 36, 3337-3344.

50 M. Asplund, T. Nyberg and O. Inganäs, Electroactive polymers for neural interfaces, Polym. Chem., 2010, 1, 1374.

51 L. Forciniti, J. Ybarra, M. H. Zaman and C. E. Schmidt, Schwann cell response on polypyrrole substrates upon electrical stimulation, Acta Biomater., 2014, 10, 2423-2433. 